\title{
A NEW MODEL FOR MANAGING CHANGE: THE HOLISTIC VIEW
}

Dr Andy Adcroft, Dr Robert Willis and Jeff Hurst

$\underline{\text { Contact Details }}$

Dr Andy Adcroft

Programme Leader

School of Management

University of Surrey

Guildford

Surrey

UK

GU2 7XH

Tel: $\quad+441483682007$

E-mail: $\quad$ a.adcroft@surrey.ac.uk

Dr Robert Willis

Principal Lecturer

Ashcroft International Business School

Anglia Polytechnic University

Chelmsford

Essex

UK

Tel: $\quad$ +448452713333

E-mail: j.r.willis@apu.ac.uk

Jeff Hurst

Director

Alternative Minds

Milton Heath House

Westcott Road

Dorking

Surrey

UK

$\mathrm{RH} 4$ 3NB

Tel: $\quad$ +441306886450

E-mail: Jeff@alternativeminds.co.uk 


\section{A NEW MODEL FOR MANAGING CHANGE: THE HOLISTIC VIEW}

\section{Dr Andy Adcroft, Dr Robert Willis and Jeff Hurst}

Key Words

- Transformation

- Holistic Models

- Rationality and Irrationality

- Event, Program, Outcome, Myth

"The model that we propose is different because it combines these elements in a way that treats organisational and strategic transformation in an holistic manner."

"Like all strategies transformation is a process; it is a collection of individual activities arranged into a sequence that has an inner logic from beginning to end."

2,960 words 


\title{
A NEW MODEL FOR MANAGING CHANGE: THE HOLISTIC VIEW
}

\author{
Dr Andy Adcroft, Dr Robert Willis and Jeff Hurst
}

Origins and Assumptions

"Heroes are made by the times" (Traditional Chinese Proverb)

Strategy really matters for a number of different reasons. Within organizations, strategy allows for the co-ordination of activities, processes and functions and provides criteria on which performance can be measured, assessed and altered. Strategy forces managers to think about the future and so allows for long term decision making to be made. If strategy is an activity that brings benefits, the problem is that strategy may be a little like beauty and the accuracy of definition may lie in the eye of the beholder. Chaharbaghi and Willis (1998), for example, found 54 different, yet widely used, definitions of strategy and so it may be reasonable to conclude that whilst we cannot accurately define it, we know it when we see it. In any case, the academic discussion of meaning is of less use to practicing managers that discussion of what the activity of strategy involves. For the purposes of this article, we will assume that the central activity common across all strategy is coping with complexity.

The complexity of strategy manifests itself in many ways. The first element of complexity lies in the fundamental nature of strategy as being concerned with the relationship between the whole of the organization and the environment in which it operates. Organizations are necessarily complex creatures. Like people, they have appearances and personalities where their true nature is defined by both. Organizations are collections of tangibles like products, functions, processes and finances but they are also defined through their intangibles like cultures and sub-cultures, knowledge and learning. Similarly, environments are complex and a collection of forces (for example, economic, social and technological), actors (such as customers, suppliers and regulators) and behaviours (for instance consumer and competitor).

If the arena in which strategy takes place makes life difficult for the strategist, then the activity itself is also difficult and one of the main reasons for this is that strategy has to marry elements of both rationality and irrationality. For our purposes, rationality is considered in the traditional sense of decision making and interpretation based on reasoning where the emphasis is on hard facts, common sense and logic. By irrationality we do not mean something that is unreasonable or unsupportable but rather elements that are beyond reason such as the instinct and gut feeling of a manager that a certain course of action is the right thing to do. Similarly, the interpretation of strategic events may not always be based on rational considerations but rather may be based on the individual experiences and prejudices of those making the interpretation be they senior managers, shop floor workers, customers or industry regulators. 
If we accept that strategy is a complex business then we can also accept that there may be a use for models of strategy which allow managers to make sense of that complexity. In generic terms, the test of any good management model is, first, the extent to which it allows for more informed management decision making and, second, the extent to which its use can lead to improved organizational and business performance. In meeting this kind of broad purpose, management models can play a number of important roles. For example:

- Models can simplify complexity and in doing so clarify thinking about what has happened and what should happen next;

- Models, especially models of a strategic nature, should identify issues which are important rather than issues that may be simply interesting;

- Models can help communicate key issues of strategy to both organizations and the outside world;

- Models can provide explanations about strategy and the consequences of strategy to a variety of internal and external stakeholders.

However, we should recognise that most management models do not describe the real world, instead they provide an approximation of the real world. In this context, one of the main problems with the use of models is that, in reducing the real world into a series of fundamental approximations, the outcome is often either too black or too white because they focus on either rationality or irrationality. The model that we propose is different because it combines these elements in a way that treats organisational and strategic transformation in an holistic manner.

To understand the nature of this model, we need to think about two issues. The first issue is origin and where the model comes from. The second issue is the assumptions upon which the model is based. Origins first. Like many of the more recent ideas in management, the origins of this model lie in other disciplines. It is an example of management learning, one of the key skills required of the successful transformation agent. The model brings together two distinct theories: theories of political revolutions developed by Peter Calvert in the 1970s and theories about the importance of speech developed more recently by John Sillence.

In considering political revolutions, Calvert's approach is based around explaining what has happened. It is, in essence, post rational. Like all types of revolution, complexity is the central issue. Revolutions are distinguished by their causes, the manner in which they are carried out and the results of the revolutionary activity. Complexity is layered onto complexity because different processes of analysis will always lead to different conclusions. Similarly, to understand words, Sillence argues we must recognise that what is said will involve much more than just the words. Whether we are listening to a politician making a speech or reading a chief executive's annual report we need to ask some questions; Why did they say this? What did they hope to achieve in saying this? What did they say? What do we think it means? 
Because models are about understanding and not necessarily accurate representation, all models will be built on a series of assumptions. In economics, for example, many models begin with the assumption of ceteris paribus, other things being equal, where attention is paid to just one or two variables. In this model, there are four assumptions:

- Assumption \#1: we live in revolutionary times.

Every teenager believes that they discovered sex and every generation believes that its time is more dynamic and dramatic than others which preceded it. We would draw attention to three distinct characteristics and drivers of the current age which does make this one different. First, technological advance in areas like information technology and communications drives ever more rapid and dynamic change. The free flow of information, which is facilitated by this technological change, means that those organizations which are able to adapt and utilise it are able to secure advantage into the future. Second, the leitmotif of our age, globalization, as a process of internationalization which leads to, among other things, the creation of a global economy. Finally, speed. The faster pace of the world means that we demand ever faster outcomes, quick and instant fixes and results today.

- Assumption \#2: only those organizations whose activities are in harmony with their environments can hope to prosper.

Our second assumption, which follows on from the first, is not necessarily specific to our model. The assumption is based around adaptation and argues that in industries, sectors or markets which are subject to dynamic change, the only option for organizations is transformation and revolution.

- Assumption \#3: organizations which transform require, and will have, very different characteristics to those which stay the same.

Our penultimate assumption considers specifically the organization which must transform. We assume a degree of economic rationality and an absence of management desire to do nothing but sit around and wait for an inevitable, slow and lingering death. Our argument is that organizations which wish to transform must recognise two key ingredients of that transformation process. First, the set of skills, competencies and tools required of a transforming organization are fundamentally different to those required of an organization that either does not want to or does not need to transform. Second, there is a clear cultural component of transformation; only those organizations which have a culture, or a set of sub-cultures, which embrace dramatic change can hope to be successful.

- Assumption \#4: without knowledge, transformation can be neither implemented nor understood.

Finally, we assume the necessity of understanding. We would argue that any story of transformation will be unique to the organization involved. Be it in terms of triggers, processes or outcomes, no two stories will be the same. However, where there will be similarity is in the complexity of the undertaking which must be understood before, during and after the transformation. For us the first step in the transformation may not be about setting objectives but 
rather gaining an understanding of the complex web of activities, processes, stakeholders and so on which must be involved and how the results may ripple out to the whole organization.

The Model

"A bridge never crossed is a life never lived" (Traditional Chinese Proverb)

Like all strategies transformation is a process; it is a collection of individual activities arranged into a sequence that has an inner logic from beginning to end. This model suggests that to understand transformation there are 4 key points at which analytical interventions are necessary. These points of intervention are:

- The Transformation Event: why the transformation happened and what was intended to come out of the transformation as a whole;

- The Transformation Programme: how transformation occurs, the key management decisions and activities through which change is (or was) realised;

- The Transformation Outcome: the nature of the transformed organization, what resulted from the first two stages;

- The Transformation Myth: not what happened, but how the transformation is interpreted and understood by both outsiders and insiders.

Diagrammatically, the model can be represented as follows:

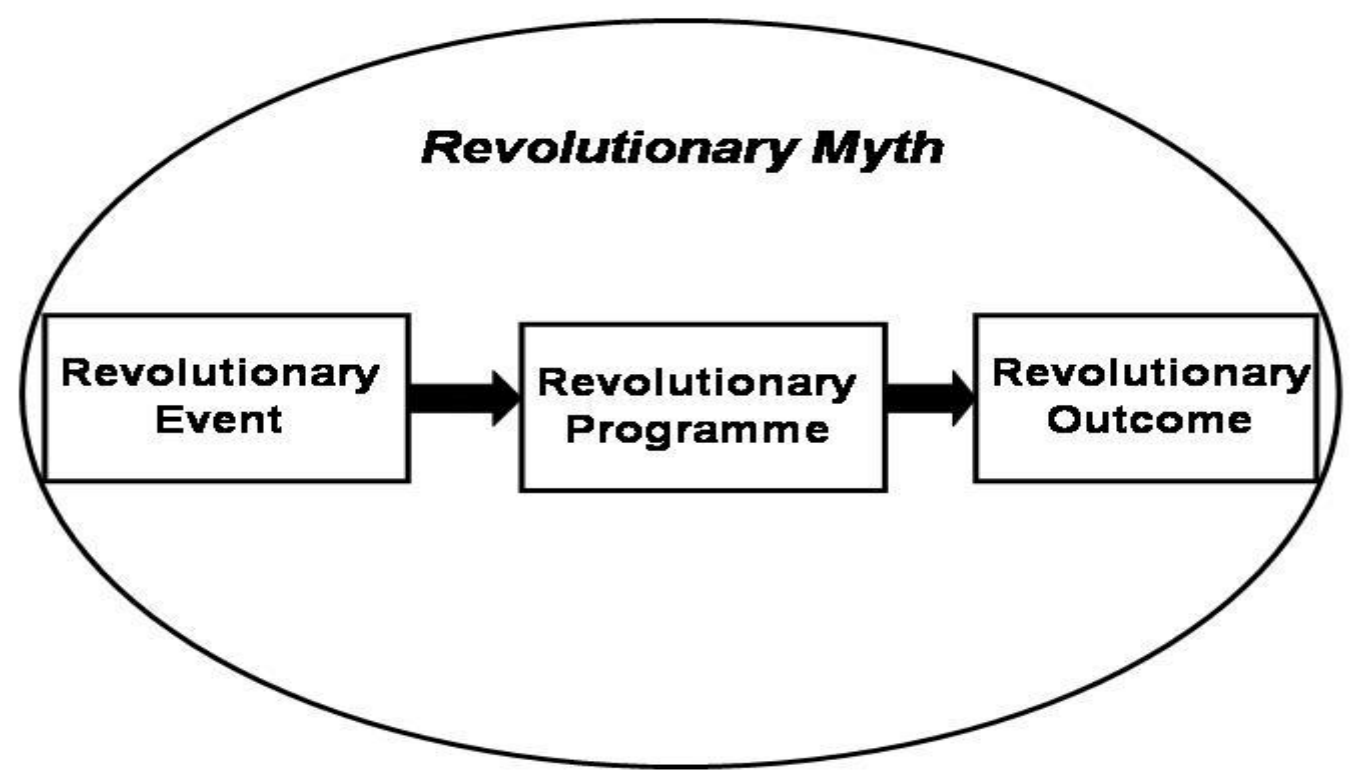

The core of the model is made up of the three key points of event, programme and outcome which are driven by the principle of consistency. It is built around the notion that all the activities in any change process must be coherent with one another. It allows for post rational enquiry and understanding of what has been done and why it may have been problematic. It allows for ongoing 
assessment of a change process that is underway and it allows for the planning and preparation of change process yet to begin. All of this is rational; the process is logical and represents common sense as much as excellent or innovative management. This form of rationality, however, can only take understanding so far and thus we have the fourth point, the transformation myth. This element deliberately stands away from the core process for two reasons. First, it is intended to reflect the view that any change process will be influenced by not just what is happening but also what is thought to be happening, how events are understood. Second, it reflects the point that, like strategy, the outcome of transformation is open to any number of different interpretations based around the peculiar characteristics of the stakeholder trying to get to grips with a process that may have finished. We now consider each of these components in turn.

\section{The Transformation Event}

"Go not ahead with nothing in front" (Traditional Chinese Proverb)

Organizations do not undergo a process of transformation by accident; unless managers make clear and deliberate decisions to transform, things will stay the same or the organisation will wander aimlessly into the future. This is the starting point for the analysis and involves some important questions. Why did this happen? Why should this happen? Central to the success of any change program is an understanding of the likely repercussions of the transformational event.

The event itself can be all manner of things. It could result from a senior manager hearing of a new concept in the industry, an unexpected result on the bottom line, the failure of a new product, a loss in market share or the entrance of new competition. Analysis needs to go beyond the simple event itself and the transformation agent needs to think about the underlying causes. Thinking about why something must happen allows the process of deciding what should happen to begin, especially a consideration of what the transformation should deliver, the clear outcomes the organization hopes to achieve.

For many organizations, a change program is only likely to give desired results if it is planned and directed. For some this will be a top-down process and for others it may be more organic. Perhaps the central point is that change must involve many stakeholders within the organization be they instigators or drivers of change or simply those who are affected by the change. Whatever the case, change is more likely to fail when the reasoning is poorly communicated and hence understood.

\section{The Transformation Program}

"Who cannot sail a ship when the sea is calm" (Traditional Chinese Proverb)

The hardest part of any transformation process is knowing where to begin, Mao Tse Tung said that every journey begins with a single step but taking that first step into something new and different is often the hardest step of all. More questions. Is the way we are going to change consistent with our 
objectives? Does this program of change address the issues which triggered it in the first place? Transformations and revolutions are always unpredictable so is our program clear enough that everyone will know we are serious and flexible enough that we can adapt it along the way?

The problem with programs in this context is that there are no generic, off the shelf answers which have universal applications across all organizations and businesses. Every organization is different and so by definition each transformation process must be different. They may all have the same components, but there are always many nips and tucks needed. Planning, adaptation, first steps and so on may be common to many programmes, but what are the key issues in developing and analysing a transformation? How important is engagement and communication? Do all stakeholders have to buy-in and, if so, how can this be achieved?

\section{The Transformation Outcome}

"Flowers cannot remain red for a hundred days" (Traditional Chinese Proverb)

Knowing when to stop, or when you have reached the desired outcome, is often difficult to determine. Models often provide more questions than answers. Where has this journey taken us? Has our transformation made us better or just different? Have we transformed in the way we needed to? Have we met the objectives we set ourselves? Have we dealt with the threats that started this process? Which opportunities have we seized? Have we changed enough? Have we changed in the way we expected to? If not, why not? What lessons have we learnt? Does change ever stop? Are the most successful transformations those whose outcome is a culture where a constant desire to improve, even on a small scale, has been created?

\section{The Transformation Myth}

"A man thinks he knows" (Traditional Chinese Proverb)

What people believe has happened can be a major factor in success or failure. For example different stakeholders will have different perceptions and create their own myths about what they see or experience. Different stakeholders will have a different relationship and expectation with and of the transforming organizations and their attitudes and behaviour will be governed by this. Customers may look at a transformed organization and judge success on whether it delivers the price or quality they want whereas employees may create their judgements around perceptions of job satisfaction or security. What is seen by a rational, desired outcome for some may be seen as the biggest mistake the organisation can make by others. Behaviour is often governed by our perceptions of events rather than the mechanics of the events themselves.

Value

"Practice makes for true knowledge" (Traditional Chinese Proverb)

Transformational strategies are often like weather forecasting. In the depths of winter we know that a few months down the line the snow and wind will be 
replaced by the hot sun but even with all the technology available we can never be much more sure than that. We know it will be warmer but we cannot predict the specific weather on a specific day in the future. With strategies aimed at transforming the nature of organizations all that can be certain is that the future will be different to the past. Our argument is that in coping with uncertainty, models such as the one in this article which provide holistic overviews through combining rational and irrational elements, can offer value in a number of ways:

- It allows for systematic, post rational analysis of an experience of transformation undertaken. People in general, and managers in particular, learn best when they are active learners and reflect on their own experiences. This kind of management learning is best facilitated through a process which combines structure with rigour;

- It allows for an understanding of why transformations fail. Successful transformation is based on a series of different activities combined into a coherent process; without coherence failure is inevitable and without a way of understanding such lack of coherence we will never know why it went wrong;

- It allows for an appreciation of the possibilities of transformation. It provides a process of pre-action analysis which determines what can and cannot be done and where the priorities for management intervention and focus must lie.

\section{Sources}

Calvert, P. (1970) A Study of Revolution, Clarendon Press, Oxford, UK. Chaharbaghi, K. and Willis, R. (1998) Strategy: The missing link between continuous revolution and constant evolution in International Journal of Operations and Production Management, Volume 18, Number 9/10.

Sillince, A. A. (1999) The role of political language forms and language coherence in the organizational change process in Organisational Studies, Volume 20, Issue 3. 